\title{
A Modified Transfer Matrix Method for Modal Analysis of Stepped Rotor Assembly Applied in the Turbomolecular Pump
}

\author{
Yiming Zhang, ${ }^{1,2}$ Jiqiang Tang, ${ }^{2,3}$ and Tong Wen $\mathbb{1}^{2,3}$ \\ ${ }^{1}$ Beijing Engineering Research Center of High-Speed Magnetically Suspended Motor Technology and Application, \\ Beihang University, Beijing, China \\ ${ }^{2}$ Ningbo Institute of Technology, Beihang University, Ningbo, China \\ ${ }^{3}$ Research Institute for Frontier Science, Beihang University, Beijing, China \\ Correspondence should be addressed to Tong Wen; wentong@buaa.edu.cn
}

Received 31 August 2021; Revised 25 December 2021; Accepted 5 January 2022; Published 27 February 2022

Academic Editor: Yuanping XU

Copyright (C) 2022 Yiming Zhang et al. This is an open access article distributed under the Creative Commons Attribution License, which permits unrestricted use, distribution, and reproduction in any medium, provided the original work is properly cited.

\begin{abstract}
An accurate modal analysis of the stepped rotor assembly is significant in the design process of rotors. The transfer matrix method (TMM) is widely used in the modal analysis of the rotor. However, the influences of the step and the assembling modes are not considered in the traditional TMM. In this paper, a modified transfer matrix method is presented for the modal calculation of the rotor in the turbomolecular pump. The stiffness reduction of stepped segments and the effect of assembled components are considered in this modified method. First of all, the traditional transfer matrix model is built. Then, the stepped segments of the rotor are changed into conical segments and the lumped mass model of conical segments is calculated. Next, the conicities of conical segments are analyzed and relations between conicities and dimensions of the rotor are discovered. Finally, the stiffness factors which can describe the effects of different assembling modes are introduced and optimized. The optimized stiffness factors are analyzed and explained from the perspective of contact stress. The modal of the stepped rotor assembly could be computed with high accuracy by using this method.
\end{abstract}

\section{Introduction}

High-speed motors show the advantages of high-power density, small volume, and small moment of inertia, which leads to extensive applications in the air blower, air compressor, and molecular pump, etc. [1]. However, the increase of the speed brings higher requirements for the static and dynamic analysis of the rotor. Though the application of the magnetic bearings can suppress the vibration of the rotor through different active control $[2,3]$, the dynamic design is still significant in the process of the rotor design. High speed may change the state of the rotor into a flexible state, which intensifies the complication of control. For the rigid state of the rotor, the first-order bending frequency should be larger than the rated speed with a margin of safety. Furthermore, the optimization is usually required in the rotor design process and the rotor modal is one of considerations. Finally, the accurate analysis of the rotor dynamics is conducive to the control analysis and unbalanced response analysis. Therefore, one of the purposes of dynamic analysis is to establish the accurate dynamic model for different shapes of the shaft. For example, the viscoelasticity of the material was considered and the force vibration analysis of viscoelastic helical rods with varying cross section and functionally graded material was investigated in [4]. Also, the uniform formulation of the dynamic vibration analysis of multispan beams was presented by using an efficient domain decomposition method and the vibration characteristics was obtained by using Rayleigh-Ritz method [5]. The main purpose of them is to establish a more accurate dynamic model.

Plenty of research contributes on the dynamic analysis. There are two major methods: finite element method (FEM) and transfer matrix method (TMM). In FEM, rotors are divided into the small elements and analyzed through the FEM software, which has the advantage of high accuracy [6]. 
The dynamic characteristics of the misaligned rigid rotor system were analyzed based on FEM [7]. However, the FEM analysis usually consumes considerable computation resources and time, especially for the nonlinear contact analysis, and it is inconvenient to change parameters of the system. This is unsuitable for the rotor optimization in the design process. TMM was first proposed by Prohl [8]. In TMM, the rotor is divided into several segments, and the state vectors of the adjacent segments are connected by the transfer matrix. The size of the matrix in TMM does not enlarge with the increment of rotor segments. And the accuracy of results is irrelevant to the quantity of rotor divisions. Thus, TMM is suitable for the modal analysis and optimization of the rotor with the advantages of simplicity of program and less consumption of time. Nevertheless, the numerical instability of the Prohl TMM will occur with the increment of the order of frequencies. Hence, Horner and Pilkey proposed the Riccati TMM to improve the numerical stability [9]. TMM can achieve similar accuracy to FEM on the modal calculation of the rotating body. Many researchers have studied the rotor modal analysis using the traditional TMM or made some modifications [10-15]. Also, the combination of FEM and TMM can achieve a better result. The FEM was incorporating into the transfer matrix and the transfer symplectic matrix was derived based on the variational principle and the numerical accuracy is better than that of the traditional method [16].

The rotor usually possesses multiple steps to provide shoulders to support rings or rolling bearings. These steps constitute abrupt changes of the dimensions and may cause the stress reduction [17]. The stress reduction in the steps changes the strain field of the stiffness of the rotor, which may affect the modal of the rotor. This change is not properly described in the mathematical model of beam theories. Some scholars considered this influence and modified modal analysis model to reckon it in [18-23]. The analytic framework was developed to study the double-beam systems and this analytic framework could calculate the exact natural frequencies of the discontinuous rotor [18]. The free vibration analysis of multiple-stepped beams was conducted by the discrete singular convolution method and the Adomian decomposition method, respectively $[19,20]$. The multistepped rotor systems were analyzed using a new method called continuous segment method [21]. All this literature solves the dynamic analysis of the stepped rotor by the numerical methods. However, the traditional TMM does not consider the effect of steps. Thus, an equivalent physical model of the stepped rotor needs to be developed and applied in TMM to calculate the modal of the stepped rotor with higher accuracy.

Besides, the rotor is normally designed as an assembly which consists of the rotor shaft and some components. These components also provide stiffness and mass for the rotor, which affects the modal of the rotor. Therefore, many scholars have also studied the assembly. Some focus on the methods of the assembly sequence which are usually used on the automated assembly. The novel automated method was proposed and developed to solve the disassembly sequence planning problem [24]. A novel stability concept for subassembly detection was presented to minimize the complexities in PASP [25]. The other researchers focus on the effects of the assembled components to the dynamic characteristics. The modal analysis of the rod-fastened rotor was studied and the flexural stiffness of the contact interface considering the pretightening force and bending moment was derived [26]. The contact relations between components and shaft were researched by optimizing the normal contact stiffness factor in the finite element model [27]. An equivalent local modulus of elasticity was optimized to reflect the influence of the interference fit between the shaft and the components. Various cases of interference were considered [28, 29]. The rotor assembly with a shrink-fitted joint was studied using the three-dimensional solid finite element approach. Meanwhile, the contact behaviors of various interference at different rotational speeds were analyzed [30]. These references focus on the interference fit of the rotor assembly and stiffness contributions of the other assembling modes, such as clearance fit and screw joint, are not considered.

This paper mainly proposes a modified TMM which includes the effects of the abrupt change and assembling modes of the stepped rotor assembly. This method is utilized to calculate the modal frequency of the rotor assembly applied in the turbomolecular pump. First, the influence of the stress reduction of the stepped shaft is taken into consideration. The step segments are replaced with conical segments and the equivalent model of conical segments is calculated. Next, conicities of conical segments are optimized. The relations between the conicities and dimensions of conical segments are analyzed. Then, the equivalent lumped mass model of the rotor assembly is established and influences of different assembling modes are revealed by introducing stiffness factors. Finally, stiffness factors are optimized with the target of modal test. Optimized stiffness factors are analyzed and explained from the perspective of contact stress. The modal of the stepped rotor assembly could be computed with high accuracy by using this modified TMM.

\section{TMM for the Modal Analysis of the Rotor}

\subsection{The Structure of the Rotor Assembly in the Turbomolecular} Pump. A turbomolecular pump with pumping speed $700 \mathrm{~L} /$ $s$ is designed and the rated speed of the rotor is $42000 \mathrm{rpm}$. For requirements of the ultra-clean environment and the ultra-high vacuum, the magnetic bearing technology is chosen for the rotor. The main components include the rotor assembly, pump blade, stators of magnetic bearings, and stator of motor.

Modal analysis is a significant part in the stage of the pump design. The rotor assembly in the turbomolecular pump is the first part to be designed and analyzed. The model of the rotor assembly is shown in Figure 1. This rotor assembly is applied in the turbomolecular pump which is supported by magnetic bearings. The rotor assembly is composed of the shaft, permanent magnet, locating rings, sleeve, nut, and magnetic bearing rings. The modal frequency is analyzed by the TMM. In order to 


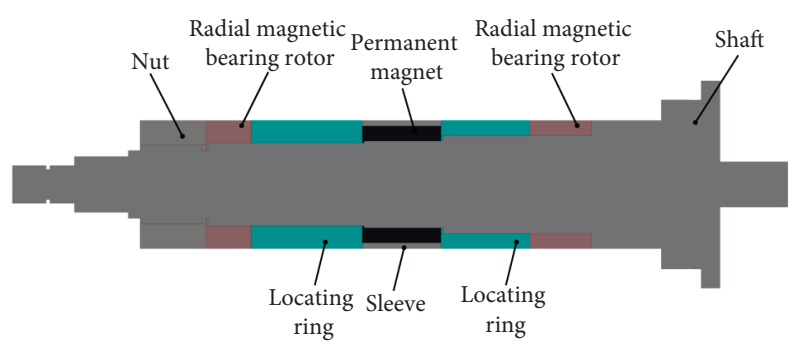

FIgURE 1: Model of the stepped rotor assembly.

improve the accuracy, the modified TMM is proposed in this paper. Effects of the abrupt change and different assembling modes are considered during the analysis. First of all, the model of the TMM needs to be established.

2.2. The Traditional TMM Model of the Rotor. The traditional TMM is based on the lumped mass model, which is composed of alternately connected disks with lumped mass and flexible shafts without mass. There are four steps for calculating frequencies of the rotor by TMM. First, the rotor is separated into several segments, which are usually the cylindrical segments. Second, each segment is equivalent to a no-mass shaft with two thick disks at the end of the shaft. Third, the lumped mass models of the segments are connected together. The mass and moment of inertia on the node between the adjacent segments are the sum of the mass and moment of inertia of the disks at the adjacent end of the segments. Finally, the transfer matrix is obtained and solved and the frequencies are calculated. The basic element of the lumped mass model is a thick disk with a no-mass shaft and the external support is located on the disk. Figure 2 shows the basic element of the lumped mass model. The state of the section of each element is expressed by four parameters, and the relations of adjacent section are established by the way of a transfer matrix. The four parameters can be expressed with the following vector [10]:

$$
\{Z\}_{i}=[x, \alpha, M, Q]_{i}^{T},
$$

where $\{Z\}_{i}$ is the state vector of the $i$-th section. $x_{i}, \alpha_{i}, M_{i}$, and $Q_{i}$ are the displacement, slope of the axis, moment, and shear force of the $i$-th section, respectively.

The transfer matrix for the cross section of the disk is obtained according to the D'Alembert's principle, and the transfer matrix for the cross section of the shaft is obtained through the principle of the Timoshenko beam. Two parts are combined and the transfer matrix of each segment is achieved as follows:

$$
Z_{i+1}=[\mathbf{B}]_{i}[\mathbf{D}]_{i} Z_{i}=[\mathbf{T}]_{i} Z_{i}
$$

where $[\mathbf{B}]_{i}$ is the transfer matrix of the $i$-th shaft segment and $[\mathbf{D}]_{i}$ is the transfer matrix of the $i$-th disk segment. Two matrices are concretely expressed as

$$
\begin{aligned}
& {[\mathbf{B}]_{i}=\left[\begin{array}{cccc}
1 & l & \frac{l^{2}}{2 E I} & \frac{l^{3}}{6 E I}(1-v) \\
0 & 1 & \frac{l}{E I} & \frac{l^{2}}{2 E I} \\
0 & 0 & 1 & l \\
0 & 0 & 0 & 1
\end{array}\right]_{i}} \\
& {[\mathbf{D}]_{i}=\left[\begin{array}{cccc}
1 & 0 & 0 & 0 \\
0 & 1 & 0 & 0 \\
0 & \left(J_{p}-J_{d}\right) \omega^{2} & 1 & 0 \\
m \omega^{2}-K & 0 & 0 & 1
\end{array}\right]_{i}}
\end{aligned}
$$

where $E$ is the modulus of elasticity. $I$ is the section moment of inertia. $l$ is the length of the segment. $v=6 E I /\left(k_{a} G A l^{2}\right)$ is the shear coefficient of the cross section. $k_{a}=0.886$ is the coefficient dependent on the shape of the cross section. $G$ is the rigidity modulus. $A$ is the cross-sectional area. $J_{p}$ and $J_{d}$ are the polar moment of inertia and diametric moment of inertia, respectively. $m$ is the mass of the segment. $K$ is the stiffness of the support. $\omega$ is the critical speed of the shaft.

The transfer matrix of the whole rotor is obtained by multiplying the transfer matrix of each segment. The parameters of the cross section at both ends of the rotor can be obtained according to the different states. For example, when the rotor is in the free state, the moment and shear force of the section at both ends of the rotor are zero. Then, the system of homogeneous linear equations is obtained and $\omega$ is the variable. The determinant of this system should be zero. Finally, the critical speed of the rotor can be calculated by solving the determinant based on the Riccati method.

\section{The Modified TMM considering the Effect of the Stepped Shaft}

3.1. The Influence of the Stepped Shaft. The lumped mass model in TMM is established according to the Timoshenko beam theory, and each segment is regarded as the cylindrical shaft. Generally, the rotor possesses multiple steps to provide shoulders to support rings or rolling bearings. The stress reduction in the step is ignored in the traditional TMM, which leads to the higher frequency of bending modes. This can be proved with a simple model as follows.

Figures 3(a) and 3(b) show a cylindrical homogeneous equidiameter shaft and a stepped shaft, respectively. The density of shafts is $7850 \mathrm{~kg} / \mathrm{m}^{3}$. The modulus of elasticity is 2 $\times 10^{11} \mathrm{~Pa}$. Nowadays, the modal analysis of the single shaft by FEM is mostly accurate when the elements are properly divided. Therefore the traditional TMM and FEM using ANSYS are adopted separately to calculate the modal of these shafts and the errors can be obtained. In TMM, the equidiameter shaft is divided into several segments and the length of each segment is $100 \mathrm{~mm}$ due to the simple structure. The stepped shaft is first divided into 3 parts and 


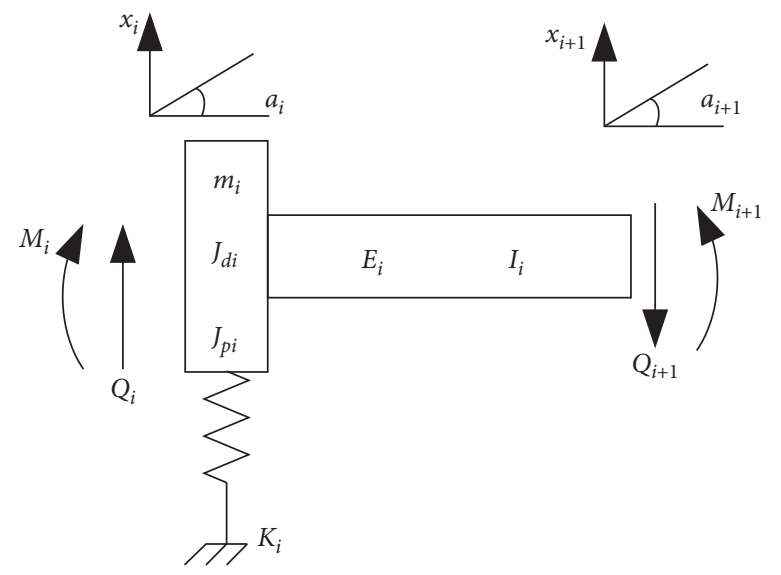

Figure 2: Model of lumped mass.

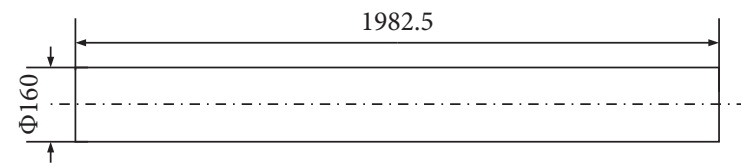

(a)

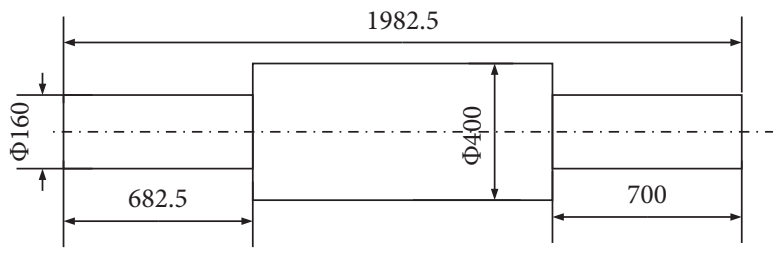

(b)

Figure 3: The simple models of rotor. (a) The equidiameter shaft and (b) the stepped shaft.

each part is an equidiameter segment. Then, each part is divided into several segments and the length is $100 \mathrm{~mm}$. In FEM, the element size of the shaft is $20 \mathrm{~mm}$ since the parameters of the shafts are large and the structures are simple. For the equidiameter shaft, the nodes and elements of the mesh are 55607 and 12978, respectively. For the stepped shaft, the nodes and elements are 32781 and 18482, respectively. The first two-order bending frequencies of two shafts are shown in Tables 1 and 2.

It can be seen that the errors of the cylindrical homogeneous equidiameter shaft between TMM and FEM are pretty small, which means TMM provides high accuracy for the equidiameter shaft. The frequencies by TMM are lower than those by FEM. The main reason is that TMM adopts the lumped mass distribution method and FEM adopts the distributed mass method.

The errors between TMM and FEM for the modal frequencies of the stepped shaft increase, and the results of TMM are larger than those of FEM. In fact, when the aspect ratio of the step or the ratio of diameters of the step increases, the error will also increase. Figure 4 shows the variation of the error between TMM and FEM when the aspect ratio or the ratio of diameters of the step increases. The reason of this error can be observed by the static analysis of the stepped shaft. Figure 5 is referred from [17] and it shows the result of distribution of the stress under the static deformation of the stepped shaft. The distribution of the static bending stress indicates that the stress in the corner is too small to be ignored. Since the stress in the corner of the step is almost zero, these regions hardly contribute to the stiffness of the shaft, which effects the deformation of the shaft in static analysis in [17]. Essentially, the deformation of the shaft in bending modal is the same as that in static analysis. That is to say, for the stepped shaft, the stress still vanishes in the corner of the step and the low-stress regions in the step will also reduce the stiffness of the shaft. The decrease of the stiffness will decrease the frequency of the bending modal, which is not considered in the traditional TMM. This is the reason why the results by TMM are higher than those by FEM in Table 2. Therefore, in order to obtain the more accurate results of the stepped shaft by using TMM, this factor should be considered, and the regions that hardly contribute to the stiffness in the stepped shaft need to be removed. In this paper, the conical segment is accepted to replace the stepped segment as the transition segment. Therefore, the regions without stress are removed. In this model, the crucial parameter is the conicity of the conical segment, which determines the regions that make less contribution to the stiffness of the shaft.

3.2. The Approach to Discretization of the Stepped Shaft. Before the TMM is applied on the shaft, the shaft needs to be separated into several segments and each segment is equivalent to a no-mass shaft and two thick disks to obtain the lumped mass model, which is shown in Figure 6(a). The left figure is the divided segment and the right figure is the lumped mass model. If the segment comprises several shafts with different diameters, it can be equivalent to the lumped mass model as well, which is shown in Figure 6(b). The lumped mass model should be transformed under the principles of lumped mass, lumped moment of inertia, 
TABLE 1: The natural frequencies of the equidiameter shaft.

\begin{tabular}{lccc}
\hline & Traditional TMM (Hz) & FEM (Hz) & Error (\%) \\
\hline 1st order bending frequency & 179.21 & 179.89 & -0.37 \\
2nd order bending frequency & 481.60 & 481.77 & -0.04 \\
\hline
\end{tabular}

TABLE 2: The natural frequencies of the stepped shaft.

\begin{tabular}{lccc}
\hline & Traditional TMM (Hz) & FEM (Hz) & Error $(\%)$ \\
\hline 1st order bending frequency & 243.97 & 226.01 & 7.95 \\
2nd order bending frequency & 411.64 & 387.88 & 6.13 \\
\hline
\end{tabular}

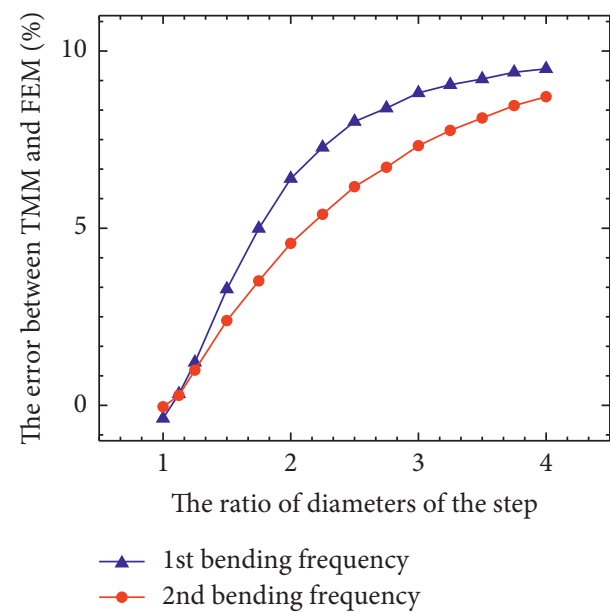

(a)

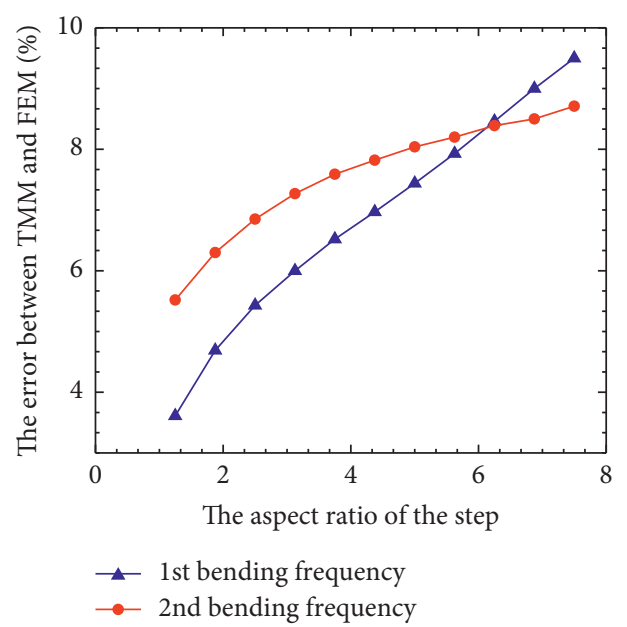

(b)

Figure 4: The variation of the error between TMM and FEM. (a) When the ratio of diameters of the step increases and (b) when the aspect ratio of the step increases.

equivalent stiffness, and equivalent deformation of shear. It means that the mass, the moment of inertia, and the section moment inertia in (3) and (4) are the equivalent parameters. However, the stiffness reduction is not considered in this transformation. In the modified TMM model in this paper, the stepped segment is replaced by the conical segment. In addition, the equivalent model of the conical segment should be calculated again with the same principles.

Similar to the cylindrical segment, the conical segment shown in Figure 6(c) is divided into two disks and a no-mass shaft. The dashed part in Figure 6(c) is the stepped part of the shaft and the conical segment is the equivalent model of the stepped part. The equivalent parameters described in the disk are mass $m$, polar moment of inertia $J_{p}$, and diametric moment of inertia $J_{d}$. The subscript $L$ and $R$ represent left disk and right disk. The equivalent parameters described in the no-mass shaft are equivalent section moment of inertia $I_{e}$ and equivalent cross-sectional area $A_{e}$. These equivalent parameters are then used in the transfer matrices in (3) and (4).

Notice that the conical segments only represent the stiffness reduction of the stepped segments; the transition segments still contribute the whole mass and moment of inertia in the modal analysis. Thus, the discrete mass and moment of inertia should be equal to the mass and moment of inertia of the corresponding cylindrical segment (the dashed part of length $l$ and diameter $D_{2}$ ). According to the principle of lumped mass, the center of mass is unchangeable after the discretization. Therefore, the mass of the disk can be described as

$$
m_{R}=m_{L}=\frac{m}{2}
$$

where $m$ is the mass of the cylindrical segment. $m_{R}$ and $m_{L}$ are the masses of the right disk and left disk of the lumped mass model in Figure 6.

According to the principle of lumped moment of inertia, the moment of inertia is unchangeable after the discretization. The polar and diametric moment of inertia of the disk can be described as

$$
\left\{\begin{array}{l}
J_{p L}+J_{p R}=\frac{J_{p}}{2}, \\
J_{d L}+J_{d R}=\frac{J d}{2},
\end{array}\right.
$$

where $J_{p}$ and $J_{d}$ are the polar and diametric moment of inertia of the cylindrical segment, respectively. $J_{p R}$ and $J_{p L}$ are the polar moment of inertia of the right disk and left disk of the lumped mass model. $J_{d R}$ and $J_{d L}$ are the diametric moment of inertia of the right disk and left disk of the lumped mass model. 


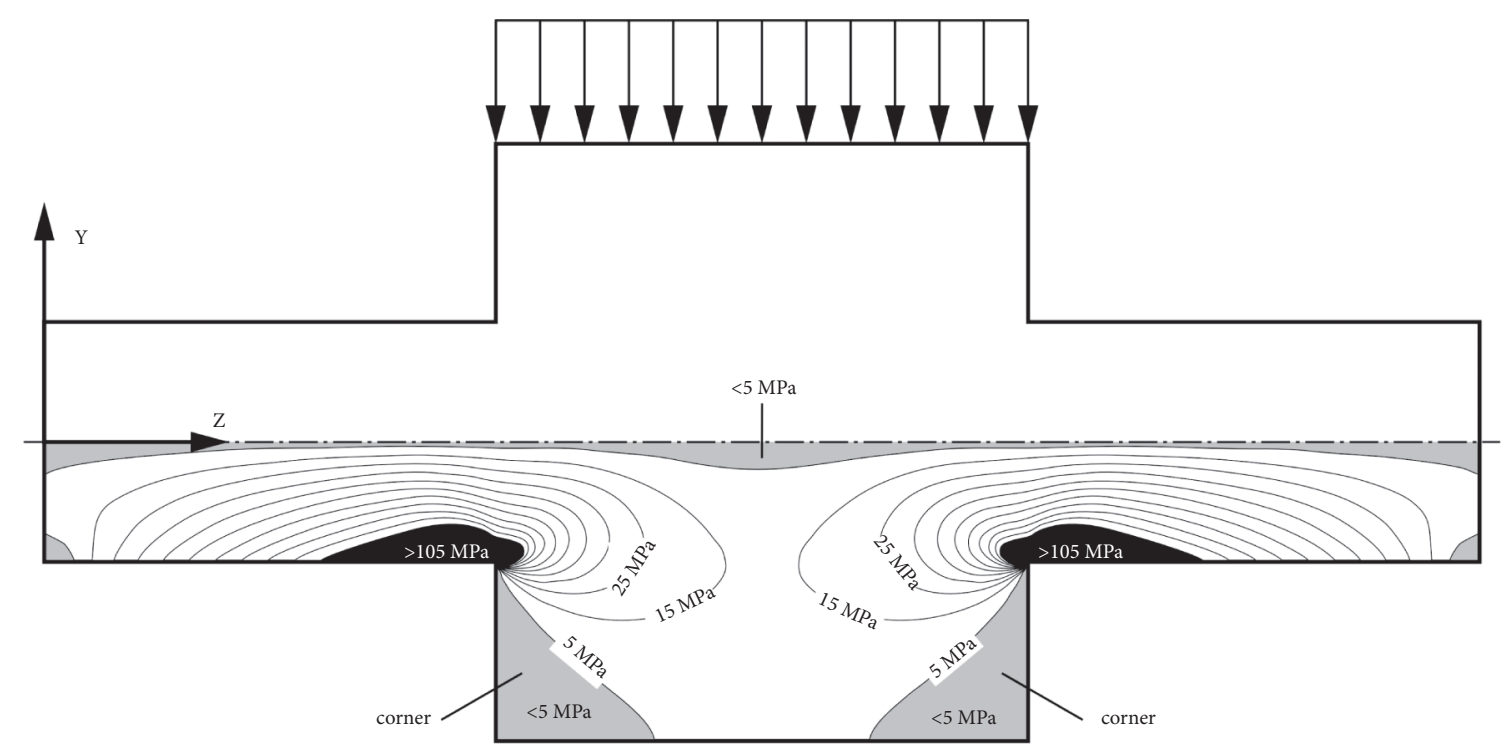

Figure 5: Distribution of $\sigma_{\mathrm{z}}$ of the stepped shaft [17].

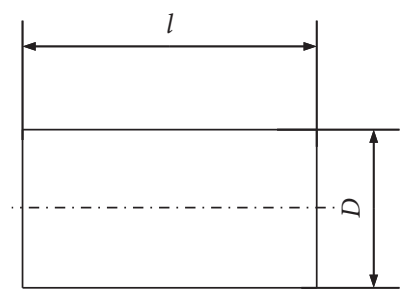

(a)

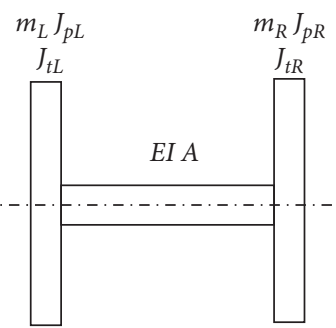

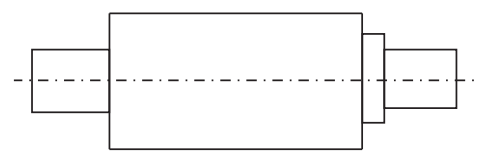

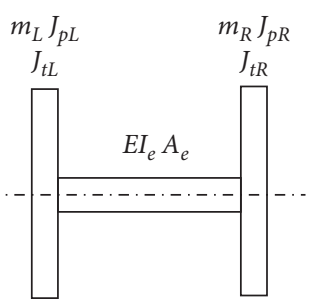

(b)

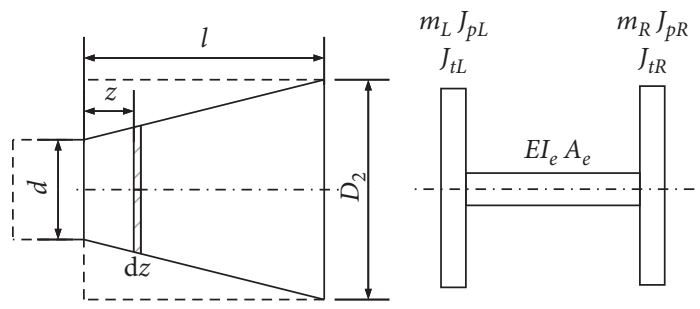

(c)

Figure 6: The lumped mass model of (a) the cylindrical shaft, (b) the multiple shaft, and (c) the conical shaft.

The equivalent stiffness of the conical segment needs to be calculated. The slopes of the cross section axis at both ends need to remain unchangeable after discretization based on the equivalent stiffness principle. A length $\mathrm{d} z$ of the conical segment is extracted, it can be regarded as cylindrical segment when $d z$ is small enough, and the slope $d z$ of length $d z$ is

$$
\mathrm{d} \theta=\frac{\mathrm{d} z}{E I(z)}
$$

The section moment of inertia of length $d z$ is

$$
I(z)=\frac{\left[z / l\left(D_{2}-d\right)+d\right]^{4} \pi}{64}
$$

where $z$ is the distance of the length $\mathrm{d} z$ from the left cross section. $I(z)$ is the section moment of inertia at length $z$.d and $D_{2}$ are diameters of the section at both ends of the conical segment.

Thus, the following equation can be obtained based on the unchangeable slope:

$$
\theta=\frac{l}{E I_{e}}=\frac{64}{\pi E} \int_{0}^{l} \frac{\mathrm{d} z}{\left[z / l\left(D_{2}-d\right)+d\right]^{4}} .
$$

Hence, the equivalent section moment of inertia of the conical segment $I_{e}$ is

$$
I_{e}=\frac{3 \pi}{64} \frac{d^{3} D_{2}^{3}}{D_{2}^{2}+d D_{2}+d^{2}} .
$$


The shear deformation should be considered in a Timoshenko beam. In the conical segment, the influence of shear deformation in bending deformation needs to remain unchangeable after the discretization. According to the deformation equation of Timoshenko beam, the influence of shear deformation on bending deformation can be expressed as

$$
\left\{\frac{Q l}{k_{a} G A_{e}}=\int_{0}^{l} \frac{Q \mathrm{~d} z}{k_{a G A(z)}}, A(z)=\frac{\pi}{4}\left[\frac{z}{l}\left(D_{2}-d\right)+d\right]^{2},\right.
$$

where $A(z)$ is the cross-sectional area at length $z$ and $A_{e}$ is the equivalent cross-sectional area of the conical segment. $Q$ is the shear force. The equations can be solved and the equivalent cross-sectional area is expressed as

$$
A_{e}=\frac{\pi d D_{2}}{4}
$$

When the conical segments of the shaft are discrete based on the above principles, the equivalent parameters $I_{e}$ and $A_{e}$ are taken into equation (4) and the transfer matrix $[B]_{\mathrm{c} i}$ of the conical shaft is expressed as

$$
[\mathbf{B}]_{c i}=\left[\begin{array}{cccc}
1 & l & \frac{l^{2}}{2 E I_{e}} & \frac{l^{3}}{6 E I_{e}}\left(1-v_{e}\right) \\
0 & 1 & \frac{l}{E I_{e}} & \frac{l^{2}}{2 E I_{e}} \\
0 & 0 & 1 & l \\
0 & 0 & 0 & 1
\end{array}\right]_{i}
$$

where $v_{e}=6 E I_{e} /\left(k_{a} G A_{e} l^{2}\right)$. The stiffness transfer matrix $[B]_{c i}$ of the conical shaft is then substituted into the traditional TMM and the modal of the stepped shaft can be obtained based on this modified TMM.

Generally, the equivalent conical segments of different steps are also different. In order to describe the conical segment quantificationally, the conicity of the conical segment $\beta$ is introduced and the relation among the conicity and other parameters in Figure 6(c) is expressed as

$$
\tan (\beta)=\frac{D_{2}-d}{2 l} \text {. }
$$

\subsection{The Relations between the Conicity and the Modal of} Stepped Shaft. For the stepped shaft, there are two common cases shown in Figure 7, according to the different situation by $b / D$ and $d / D$. The geometry of the real stepped shaft (outline) and the equivalent conical shaft (shaded) is presented. When the step is illustrated by the low $b / D$ and low $d / D$ (Figure 7(a)), the middle segment of the stepped shaft is separated into two conical segments and a cylindrical segment. When the step is illustrated by the high $b / D$ and high $d / D$ (Figure 7(b)), the middle segment of the stepped shaft is separated into two conical segments.
In order to obtain the relations between the conicity and $b / D$ and $d / D$, a series of optimizations is conducted. The stepped shaft in Figure 7 is applied and a series of $b / D$ and $d / D$ is set. The basic parameter $D$ is set to be $150 \mathrm{~mm}$. Since the density and modulus of elasticity of the shaft are independent of the conicity, they can be determined arbitrarily in this optimization. The density of the shaft is $7850 \mathrm{~kg} / \mathrm{m}^{3}$ and the modulus of elasticity is $2 \times 10^{11} \mathrm{~Pa} . b$ is selected from $10 \mathrm{~mm}$ to $190 \mathrm{~mm}$ equidistantly and the distance is $10 \mathrm{~mm} . d$ is selected from $20 \mathrm{~mm}$ to $140 \mathrm{~mm}$ equidistantly and the distance is also $10 \mathrm{~mm}$. Therefore, the range of $b / D$ is 0.067 to 1.267 and 19 values of ratios are selected. Similarly, the range of $d / D$ is 0.133 to 0.933 and 13 values of ratios are selected. Therefore, a total of 247 kinds of stepped shafts are chosen. The ranges of ratios cover the common ratio of the rotor parameters. All kinds of the stepped shafts with the same parameters of material are modeled in FEM software and the first two-order bending frequencies are analyzed. The element size of all the stepped shafts in FEM is $3 \mathrm{~mm}$ and the mesh is formed by the program automatically.

Next, the rotor models with different combination of ratios are established using the modified TMM, respectively. In TMM, the stepped shaft is divided into three equidiameter parts and then these parts are divided into several segments and the length of segments is $8 \mathrm{~mm}$. For a certain combination of ratios, the conicity is the variable in TMM model. The most suitable conicity enables the frequencies obtained by TMM to approach those by FEM. An objective function is established to calculate the error between the TMM result and FEM result:

$$
F_{s}=\min \sqrt{\left(\bar{\omega}_{1}-\omega_{1}\right)^{2}+\left(\bar{\omega}_{2}-\omega_{2}\right)^{2}}
$$

where $\bar{\omega}_{1}$ and $\bar{\omega}_{2}$ are the first and second bending frequencies by TMM and $\omega_{1}$ and $\omega_{2}$ are first and second bending frequencies by FEM, respectively.

This objective function reflects the error between TMM and FEM results, and the conicity which makes the objective function minimum is the optimized conicity under certain combination of ratios. The range of conicity is from 0 to $\pi / 2$. Since the optimization of each combination of ratios has single variable, the optimal function $f \min b n d$ in MATLAB is adopted, which is used to find the minimum of nonlinear objective with single variable. The relations among the optimized conicities, ratio of $b / D$, and ratio of $d / D$ are shown in Figure 8.

(i) When $d / D$ is unchanged, the relations between the conicity and $b / D$ can be divided into two regions. When $d / D$ is less than 0.4 , for example, in Figure 9(a), the conicity decreases first and then increases and finally slightly decreases as $b / D$ increases. When $d / D$ is greater than 0.4 , for example, in Figure 9(b), the conicity decreases rapidly and then increases slightly as $b / D$ increases.

(ii) When $b / D$ is unchanged, the variation of the conicity is mostly the same in different $b / D$. Figures 10(a) and 10(b) are the variation curve of 


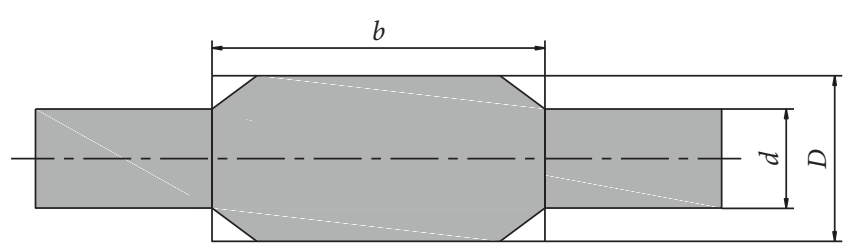

(a)

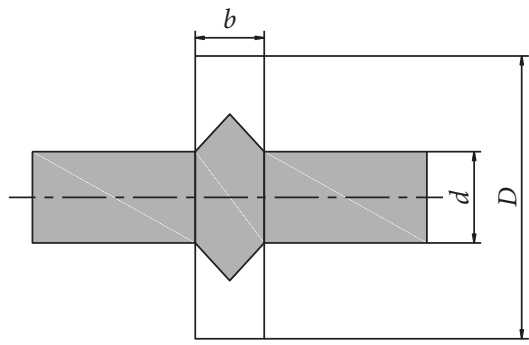

(b)

Figure 7: The modes of the stepped shaft. (a) High $b / D$ and high $d / D$ and (b) low $b / D$ and low $d / D$.

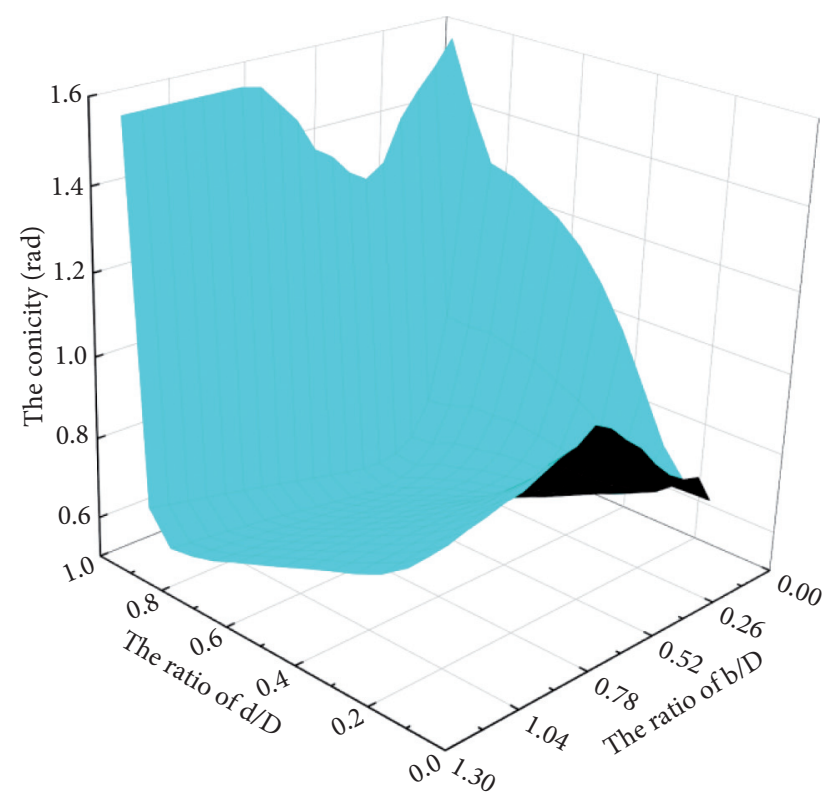

Figure 8: The relations among optimized angle and $b / D$ and $d / D$ ratios.

the conicity in different $b / D$. The conicity decreases slowly and then increases rapidly as $d / D$ increases.

(iii) When $d / D$ is greater than 0.933 , the conicity is almost close to $\pi / 2$, which means the influence of the step can be ignored.

(iv) If $d / D$ remains unchanged, when the rotor is in the state of Figure $7(\mathrm{a})$, the conicity is almost unchanged.

The results can be introduced to calculate the modal of the stepped shaft considering the effect of the step. The shaft of the rotor assembly in Figure 1 is calculated. The main dimensions of this stepped shaft are shown in Figure 11. This shaft is analyzed based on TMM and the effect of the step is considered. The conicity of each step can be obtained through the interpolation method from Figure 8. Also, the results of FEM are obtained to be contrasted. The material of this shaft is steel. The density and the modulus of elasticity are $7850 \mathrm{~kg} / \mathrm{m}^{3}$ and $2 \times 10^{11} \mathrm{~Pa}$, respectively. Considering the size of this shaft, the element size in FEM is $4 \mathrm{~mm}$ and the regions of the steps are refined. Therefore, the nodes and elements of the mesh of this shaft are 66617 and 42576, respectively. The first two bending frequencies of this shaft using the modified TMM and FEM are shown in Table 3. It can be observed that the modal of the stepped shaft is analyzed through this modified TMM model with great accuracy.

\section{The Modified TMM considering the Effects of Assembling Modes}

4.1. Modal Updating Analysis. The rotor assembly usually consists of the shaft and components which are attached by different assembling modes. Generally, there exist three assembling modes in the rotor assembly: interference fit, clearance fit, and screw joint. Different assembling modes make different contributions on the stiffness of the components, which eventually influences the rotor modal frequencies. In TMM model, the assembly segments need to be changed into the lumped mass model using an equivalent principle $[31,32]$. It can be seen from (3) and (4) that the rotor frequencies are determined by the parameters of $m, J_{p}$, $J_{d}$, and EI. Therefore, if these four parameters are unchangeable in lumped mass model, the rotor frequency will be also unchangeable.

According to the equivalent principle, the relations between the lumped mass model and the assembled segment in Figure 12 can be illustrated as

$$
\left\{\begin{array}{l}
m_{s}+m_{c}=m_{e} \\
J_{p s}+J_{p c}=J_{p e} \\
J_{d s}+J_{d c}=J_{d e} \\
(E I)_{s}+\alpha_{i}(E I)_{c}=(E I)_{e}
\end{array} \quad i=I F, C F, S J\right.
$$

where the subscript $s, c$, and $e$ represent the shaft, component, and equivalent model, respectively. In order to reflect the effects of different assembling modes, the stiffness factors $\alpha_{i}$ are introduced in (16). The subscripts of $\alpha$ mean interference fit, clearance fit, and screw joint, respectively.

According to the formula of mass and moment of inertia, the equation can be changed as 


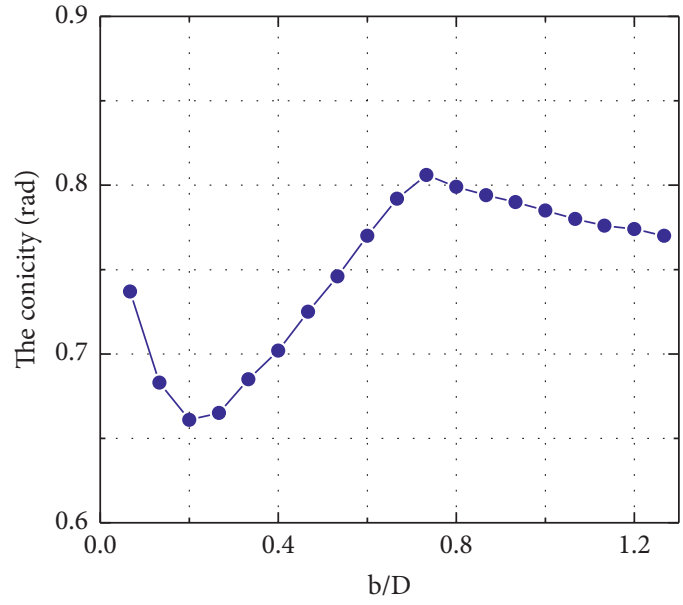

(a)

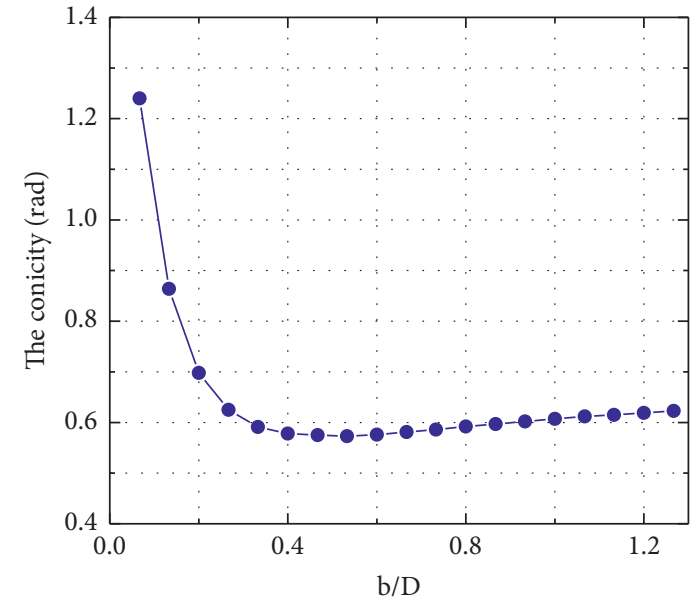

(b)

Figure 9: The conicities when $d / D$ is constant. (a) $d / D$ is 0.267 and (b) $d / D$ is 0.667 .

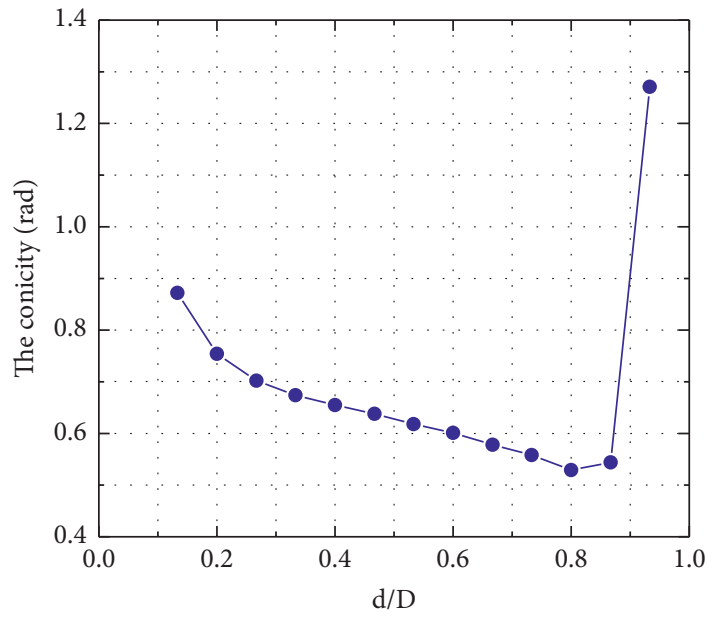

(a)

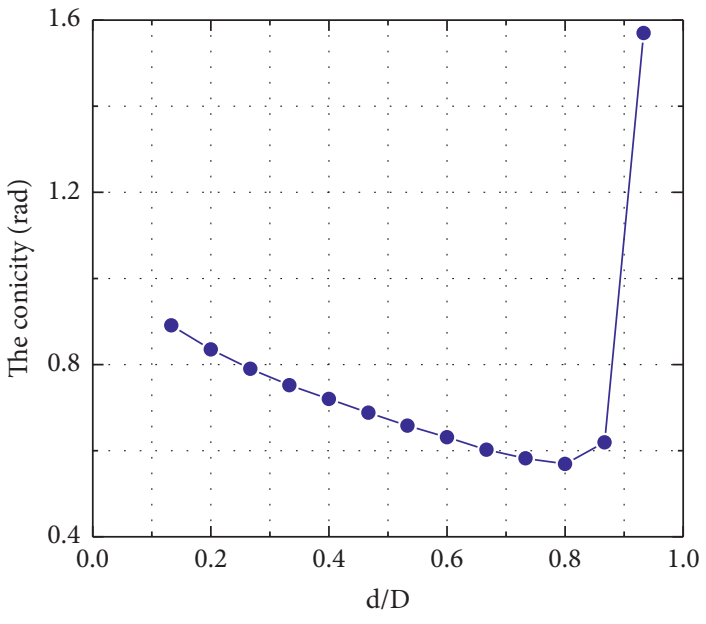

(b)

FIgure 10: The conicities when $b / D$ is constant. (a) $b / D$ is 0.4 and (b) $b / D$ is 0.933 .

$$
\left\{\begin{array}{l}
\rho_{s} r_{1}^{2}+\rho_{c}\left(r_{2}^{2}-r_{1}^{2}\right)=\rho_{e} R_{e}^{2} \\
\rho_{s} r_{1}^{4}+\rho_{c}\left(r_{2}^{4}-r_{1}^{4}\right)=\rho_{e} R_{e}^{4} \quad i=I F, C F, S J, \\
E_{s} r_{1}^{4}+\alpha_{i} E_{c}\left(r_{2}^{4}-r_{1}^{4}\right)=E_{e} R_{e}^{4}
\end{array}\right.
$$

where $\rho$ is the density. $E$ is the modulus of elasticity. $r_{1}$ is the inner diameter of the component and $r_{2}$ is the outer diameter of the component. $R_{e}$ is the diameter of the equivalent shaft. $\rho_{e}, R_{e}$, and $E_{e}$ can be solved according to these equations and the lumped mass model of the rotor assembly can be obtained.

For different assembling modes, the stiffness factors are unequal. The parameters and assembling modes of the rotor assembly in Figure 1 are listed in Table 4. On the premise of ensuring performance, the simplicity of assembly operation is considered during selecting the mode of the assembly. Therefore, the clearance fit is applied on most of parts listed in Table 4. The screw joint is applied on the nut at the end of the rotor to lock other parts. The permanent magnet is fragile and the tensile strength of the permanent magnet is small; meanwhile the compressive strength is large. Thus, the permanent magnet should be under the compressive stress when the rotor is rotated. Therefore, the interference fit is used on the sleeve to protect the permanent magnet to be under the compressive stress.

4.2. Optimization of the Stiffness Factor. A modal test is conducted for the rotor assembly in Figure 1. The test setup is shown in Figure 13(a). The acceleration sensors are glued on the rotor and connected with the power supply and the oscilloscope. The rotor is knocked by the hammer, and the vibration signal is obtained using the acceleration sensors. The modal frequencies can be calculated via the fast Fourier transform. As Figure 13(b) shows, the first two bending frequencies by modal test are $3784 \mathrm{~Hz}$ and $7599 \mathrm{~Hz}$. 


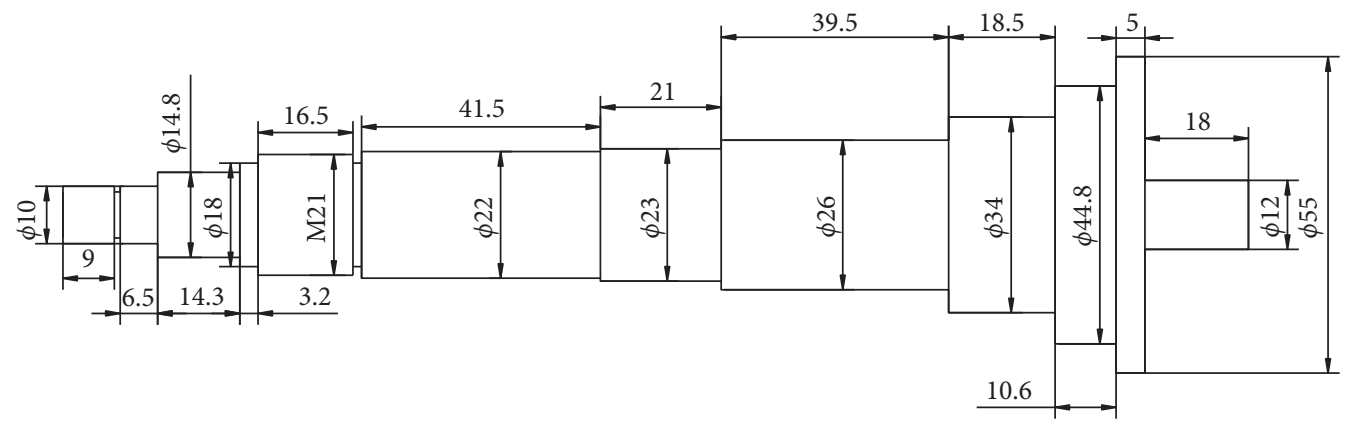

FIgURE 11: The main dimensions of the stepped shaft.

TABLE 3: The results of the rotor modal by the modified TMM and FEM.

\begin{tabular}{lccc}
\hline & Modified TMM (Hz) & FEM (Hz) & Error $(\%)$ \\
\hline 1st order bending frequency & 2837.1 & 2840 & -0.102 \\
2nd order bending frequency & 7059.6 & 7071.1 & -0.163 \\
\hline
\end{tabular}

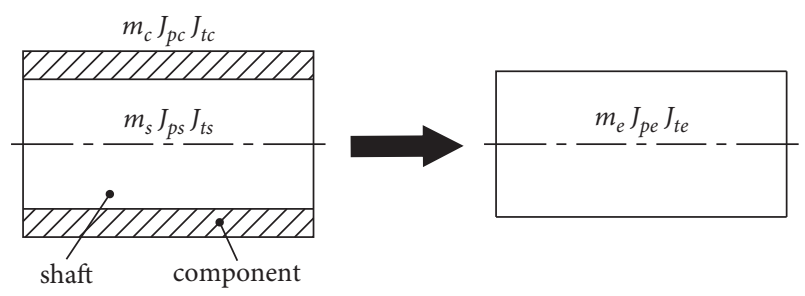

Figure 12: The rotor assembly segment and equivalent model.

In order to obtain the stiffness factors, the frequencies obtained by the modified TMM are compared with the frequencies obtained by the modal test. The most suitable stiffness factors enable the frequencies obtained by TMM to approach those by modal test. In order to search these suitable stiffness factors, the optimized method should be adopted. Particle swarm optimization (PSO) is an intelligent optimization algorithm with the advantages of easy implementation, fast convergence, guaranteed diversity, and satisfactory accuracy [33]. It can combine with the other mathematical method or mathematical model to solve some complicated problems [34, 35]. Also, with the modification of the updating method of the particle swarm, the modified PSO method can improve the local search performance and tack the multimodal multiobjective optimization problem $[33,36]$. In this paper, the optimization is single-objective. Therefore, the traditional PSO can be adopted. In order to balance the ability of the global search and local search, the variable inertia weight is adopted in PSO.

$$
w(k)=w_{\text {start } h}-\left(w_{\text {start } h}-w_{\text {end }}\right)\left(\frac{k}{T_{\max }}\right)^{2},
$$

where $w(k)$ is the inertia weight of the $k$-th iteration; $w_{\text {start } h}$ and $w_{\text {end }}$ are the inertia weight of the start and end iteration, respectively. $T_{\max }$ is the maximum number of iterations.

Next, to describe the degree of closeness of the frequencies by TMM and modal test, the mathematical model is established to be the objective function in PSO.

$$
F_{a}=\min \sqrt{\left(\bar{\omega}_{1}-\widetilde{\omega}_{1}\right)^{2}+\left(\bar{\omega}_{2}-\widetilde{\omega}_{2}\right)^{2}}
$$

where $\widetilde{\omega}_{1}$ and $\widetilde{\omega}_{2}$ are the first and second order of frequencies acquired by the modal test, respectively. The objective indicates the displacement between the frequency of TMM and the frequency of the modal test. Thus, the objective should be minimum.

$\alpha_{i}$ represents the contribution of the component stiffness. In PSO, $\alpha_{I F}, \alpha_{C F}$, and $\alpha_{S J}$ are selected to be the optimized variables, which represent the influences of interference fit, clearance fit, and screw joint, successively. The component contributes the whole stiffness when $\alpha_{i}$ is 1 and contributes part of its own stiffness when $\alpha_{i}$ is smaller than 1. Furthermore, the component contributes more than its own stiffness when $\alpha_{i}$ is larger than 1 . The constraints of the variables are listed as

$$
\left\{\begin{array}{l}
1 \leqslant \alpha_{I F} \\
0 \leqslant \alpha_{C F} \leqslant 1 \\
0 \leqslant \alpha_{S J} \leqslant 1
\end{array}\right.
$$

The generation and population of the PSO are 100 and 50 , respectively. The variation of three stiffness factors is shown in Figure 14(a) and the variation of the objective function is shown in Figure 14(b).

From the optimized variables in Figure 14, it can be summarized that different assembling modes have different contributions on the stiffness of the assembled components. The stiffness factor of the interference fit is 3.2948 , which means the component contributes more than three times of its own stiffness due to the contact stress produced by interference fit. The stiffness factor of the screw joint is 0.7522 . It shows that the component contributes part of the stiffness if assembled by screw joint. The stiffness of the clearance fit is 0.0911 , which means the component contributes very little stiffness. This is the main resource of the error of the modal analysis by FEM. In FEM, if the assembling modes are not considered and all the contacts are selected as bonded, the 
TABLE 4: The parameters and assembling modes of the rotor assembly.

\begin{tabular}{lcccc}
\hline & Inner diameter $(\mathrm{mm})$ & Outer diameter $(\mathrm{mm})$ & Length $(\mathrm{mm})$ & Assembling mode \\
\hline Radial magnetic bearing rotor & 26 & 34 & 16.5 & Clearance fit \\
Locating ring & 26 & 34 & 23.5 & Clearance fit \\
Sleeve & 31 & 34 & 21 & Interference fit \\
Permanent magnet & 23 & 31 & 21 & Clearance fit \\
Locating ring & 22 & 34 & 29.5 & Clearance fit \\
Radial magnetic bearing rotor & 22 & 34 & 12 & Clearance fit \\
Nut & 21 & 34 & 17.5 & Screw joint \\
\hline
\end{tabular}

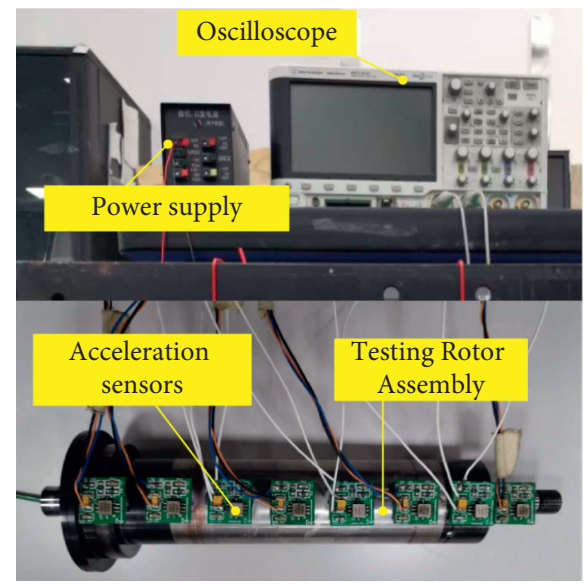

(a)
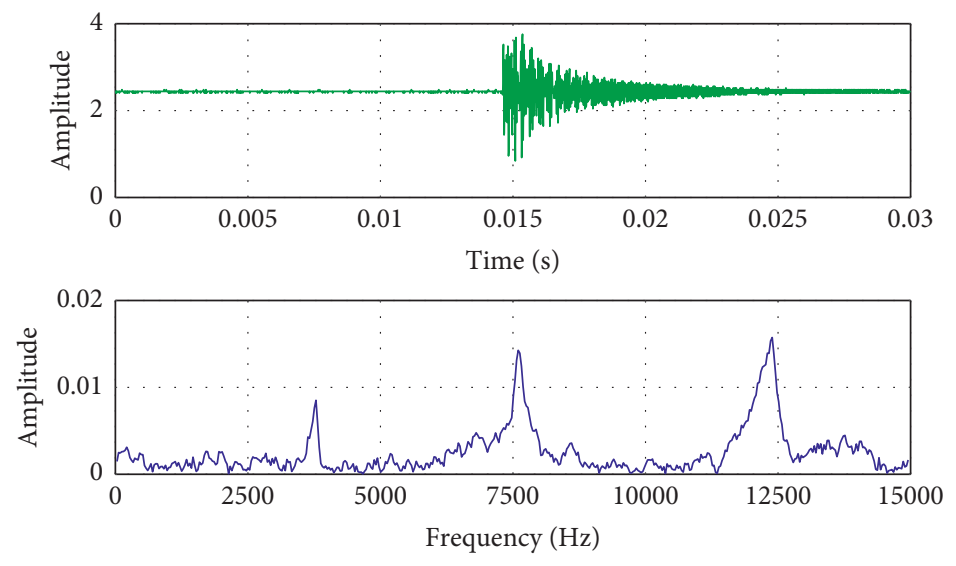

(b)

Figure 13: The modal test. (a) The test setup, and (b) the results of the modal test.

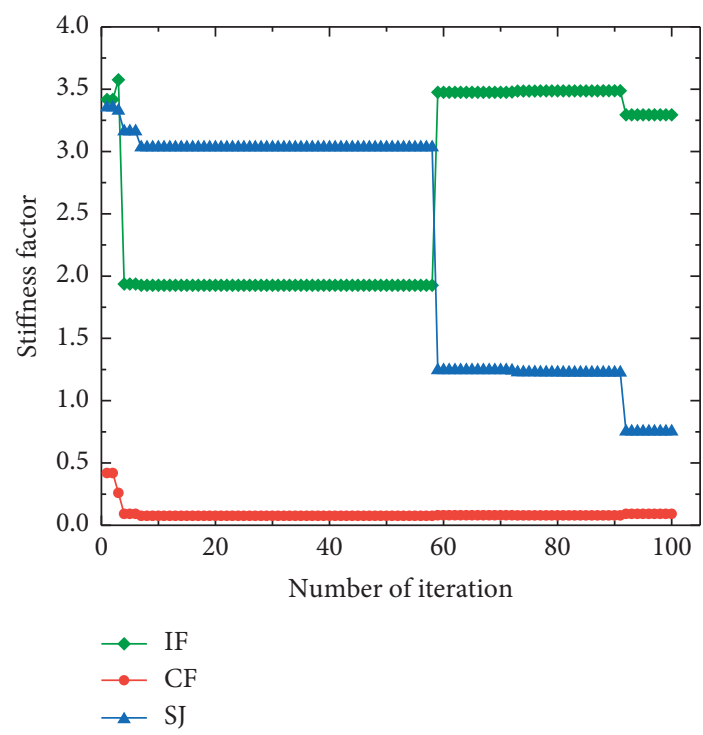

(a)

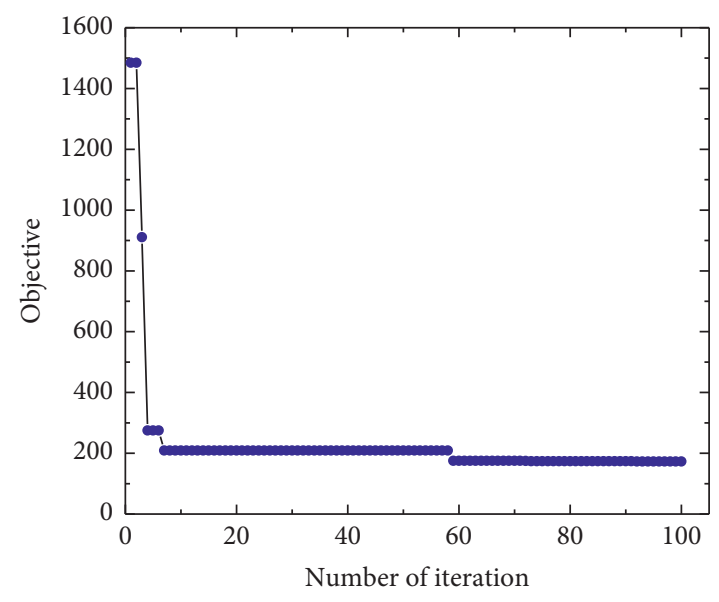

(b)

FIGURE 14: The results of the optimization: (a) the variation of stiffness factors, and (b) the variation of the objective.

results will be higher than the true results. In [6], the different contacts were selected and FKN was optimized in the modal analysis of the rotor assembly. The results of natural frequencies calculated by the modified TMM and the modal test are shown in Table 5. Moreover, the results of the modal analysis by FEM without consideration of the assembling modes and by the modified FEM in [6] are also listed as the contrast. It can be observed that the results by the FEM with bonded contacts have large errors to the modal test results. However, the results by the modified TMM are close to the results of the modal test and the results by the modified FEM. Therefore, the modified TMM can enhance the accuracy of the modal analysis of the rotor assembly. 
Table 5: The first two frequencies obtained by the modified TMM, modal test, and FEM.

\begin{tabular}{lccccc}
\hline & Modified TMM (Hz) & Modal test (Hz) & Error (\%) & FEM with bonded contacts & Modified FEM in [6] \\
\hline 1st order frequency & 3678.86 & 3784 & -2.78 & 4545.2 & 3689.3 \\
2nd order frequency & 7461.67 & 7599 & -1.81 & 9818.5 & 7577.1 \\
\hline
\end{tabular}

\section{Advantages and Limitations of the Modified TMM}

Comparing with other literature, the proposed concept in this paper is a modification to the traditional TMM. The traditional TMM has limitations on the modal analysis of the stepped shaft and the rotor assembly. Therefore, other literature usually adopts the numerical method or FEM to solve the modal analysis of the stepped shaft and rotor assembly. This paper improves the traditional TMM and considers the influences of the step and the assembled component. Therefore, this proposed concept enlarges the application of TMM, and it can be used on the multistepped rotor or the disk-shaped rotor which has a large step. Meanwhile, this proposed concept also explains the reason of the limitation of the traditional TMM. The reduction of the step and the stiffness enhancement of the assembled components produces a large error by traditional TMM. In this paper, these factors are all considered and reflected by the conicity of the conical segment and the stiffness factor. Therefore, the modified TMM has a better accuracy on the modal analysis of the stepped rotor and rotor assembly, which can be observed from the validation of the model in this paper. Also, the advantages of the traditional TMM still exist. However, this modified TMM still has some limitations. The equivalent conical segment model is only used on the rotor with the round cross section. For the stepped beam with rectangular cross section or other shapes of the cross section, this modified TMM is inapplicable.

\section{Conclusion}

This paper presented work on the modified TMM to analyze the modal of the stepped rotor assembly. The rotor was modeled according to the lumped mass principle and calculated by the modified TMM. The influence of the step and the assembling modes of the rotor were considered. First, the stepped segments were modeled by the conical segments and the equivalent stiffness and cross-sectional area were derived. Then, the conicities of conical segments were optimized and the relationships between conicities and dimensions of the stepped rotor were found. Next, the equivalent model of the rotor assembly was established and the stiffness factors were introduced to reflect the effects of different assembling modes. Finally, an optimization method was conducted to calculate the stiffness factors. The stiffness factors of different assembling modes were obtained and explained from the perspective of contact stress.

Based on the results, the following conclusions can be drawn:

(i) The abrupt change in the step of the rotor affects the modal frequency of the rotor. This influence can be embodied by substituting the step with the conical segment.

(ii) The conicity of the equivalent conical segment is related to the aspect ratio and ratio of diameters of the step.

(iii) The assembling modes exert different influences on the modal frequency of the rotor. The influences are reflected by introducing the stiffness factors. The influences of different assembling modes are connected with the contact stress of the components.

The modified TMM model proposed in this paper can be applied to calculate the modal of the stepped rotor assembly in an efficient and effective way. This model can be also used in the optimized design of the rotor due to the low time consumption and simplicity of the variable change.

\section{Data Availability}

The data used to support the findings of the study are included within the article.

\section{Conflicts of Interest}

The authors declare that there are no conflicts of interest.

\section{Acknowledgments}

This work was supported by the National Key R\&D Program of China under Grants 2018YFB0905500 and 2018 YFB0905503 and National Natural Science Foundation of China under Grants 61773038 and 62073010.

\section{References}

[1] D. Gerada, A. Mebarki, and N. L. Brown, C. Gerada, A. Cavagnino, and A. Boglietti, High-speed electrical machines: technologies, trends, and developments," IEEE Transactions on Industrial Electronics, vol. 61, no. 6, pp. 2946-2959, 2014.

[2] J. Li, G. Liu, S. Zheng, P. Cui, and Q. Chen, "Micro-jitter control of magnetically suspended control moment gyro using adaptive LMS algorithm," IEEE/ASME Transactions on Mechatronics, 2021.

[3] Y. Xu, H. Wu, and X. Guan, "Unbalance suppression for AMB rotor system using APF-SRF algorithm," Shock and Vibration, vol. 2020, Article ID 2606178, 10 pages, 2020.

[4] F. F Calim and Y. C Cuma, "Forced vibration analysis of viscoelastic helical rods with varying cross-section and functionally graded material," Mechanics Based Design of Structures and Machines, pp. 1-12, 2021.

[5] C. Gao, F. Pang, H. Li, H. Wang, J. Cui, and J. Huang, "Free and forced vibration characteristics analysis of a multispan Timoshenko beam based on the ritz method," Shock and Vibration, vol. 2021, Article ID 4440250, 18 pages, July 2021. 
[6] Z. Huang and Y. Le, "Rotordynamics modelling and analysis of high-speed permanent magnet electrical machine rotors," IET Electric Power Applications, vol. 12, 2018.

[7] M. Wang, B. Wen, Q. Han, Y. Sun, and C. Yu, "Dynamic characteristics of a misaligned rigid rotor system with flexible supports," Shock and Vibration, vol. 2021, Article ID 8876190, 16 pages, 2021.

[8] M. A. Prohl, "A general method for calculating critical speeds of flexible rotors," Journal of Applied Mechanics, vol. 12, no. no. 3, pp. A142-A148, 1945.

[9] G. C Horner and W. D Pilkey, "The Riccati transfer matrix method," Journal of mechanical design, vol. 100, no. 2, pp. 297-302, 1978.

[10] B. Han and Z. Huang, "Effective approach for calculating critical speeds of high-speed permanent magnet motor rotorshaft assemblies," IET Electric Power Applications, vol. 9, no. 9, pp. 628-633, 2015.

[11] D. S. Khamari, P. S. Kar, S. Jena, J. Kumar, and S. K. Behera, Rotordynamic analysis of high-speed rotor used in cryogenic turboexpander using transfer matrix method," in Journal of Applied Mechanics, pp. A142-A148, Springer, NY, USA, 2021.

[12] X. Rui, X. Wang, Q. Zhou, and J. Zhang, "Transfer matrix method for multibody systems (Rui method) and its applications," Science China Technological Sciences, vol. 62, no. 5, pp. 712-720, 2019.

[13] G. Chen, X. Zeng, X. Liu, and X. Rui, "Transfer matrix method for the free and forced vibration analyses of multi-step Timoshenko beams coupled with rigid bodies on springs," Applied Mathematical Modelling, vol. 87, pp. 152-170, 2020.

[14] B. Rong, X. Rui, K. Lu, L. Tao, G. Wang, and X. Ni, “Transfer matrix method for dynamics modeling and independent modal space vibration control design of linear hybrid multibody system," Mechanical Systems and Signal Processing, vol. 104, pp. 589-606, 2018.

[15] C. Feng, X. Ma, Z. Hu, and Y. Luo, "Application of an improved discretisation method for the rotor-bearing assembly in the radiated noise analysis using Riccati transfer matrix method," IET Electric Power Applications, vol. 11, no. 7, pp. 1245-1253, 2017.

[16] H. Deng, X. Fang, H. Wu et al., "Dynamic analysis of flexible rotor based on transfer symplectic matrix," Shock and Vibration, vol. 2019, Article ID 9154272, 11 pages, 2019.

[17] F. Sanchez-Marin, V. Roda-Casanova, and A. Porras-Vazquez, "A new analytical model to predict the transversal deflection under load of stepped shafts," International Journal of Mechanical Sciences, vol. 146-147, pp. 91-104, 2018.

[18] Z. Zhang, X. Huang, Z. Zhang, and H. Hua, "On the transverse vibration of Timoshenko double-beam systems coupled with various discontinuities," International Journal of $\mathrm{Me}$ chanical Sciences, vol. 89, pp. 222-241, 2014.

[19] G. Duan and X. Wang, "Free vibration analysis of multiplestepped beams by the discrete singular convolution," Applied Mathematics and Computation, vol. 219, no. 24, Article ID 11096, 2013.

[20] Q. Mao, "Free vibration analysis of multiple-stepped beams by using Adomian decomposition method," Mathematical and Computer Modelling, vol. 54, no. 1-2, pp. 756-764, 2011.

[21] A Mereles and K. L Cavalca, "Modeling of multi-stepped rotor-bearing systems by the continuous segment method," Applied mathematical modelling, vol. 96, pp. 402-430, 2021.

[22] R. M. Lin and T. Y. Ng, "Exact vibration modes of multiplestepped beams with arbitrary steps and supports using elemental impedance method," Engineering Structures, vol. 152, pp. 24-34, 2017.
[23] J. W. Lee and J. Y. Lee, "An exact transfer matrix expression for bending vibration analysis of a rotating tapered beam," Applied Mathematical Modelling, vol. 53, pp. 167-188, 2018.

[24] G. A Kumar, M. V. A. R Bahubalendruni, V. S. S. V Prasad, D Ashok, and K Sankaranarayanasamy, "A novel Geometric feasibility method to perform assembly sequence planning through oblique orientations," Engineering Science and Technology, an International Journal, 2021, In Press, Article ID S2215098621001038.

[25] A. K Gulivindala, M. V. A. R Bahubalendruni, S. S. V. P Varupala, and K Sankaranarayanasamy, "A heuristic method with a novel stability concept to perform parallel assembly sequence planning by subassembly detection," Assembly Automation, vol. 40, no. 5, pp. 779-787, 2020.

[26] Y. Liu, Q. Yuan, P. Li, and G. Zhu, "Modal analysis for a rodfastened rotor considering contact effect based on double fractal model," Shock and Vibration, vol. 2019, Article ID 4027353, 10 pages, 2019.

[27] Z. Huang, J. Fang, and K. Wang, "Dynamics of flexible rotorshaft assembly with consideration of contact behaviors," Proceedings of the Institution of Mechanical Engineers - Part C: Journal of Mechanical Engineering Science, vol. 229, no. 5, pp. 859-868, 2015.

[28] S.-Y. Chen, "An equivalent direct modeling of a rotary shaft with hot-fit components using contact element modal analysis results," Computers \& Mathematics with Applications, vol. 64, no. 5, pp. 1093-1099, 2012.

[29] S.-Y. Chen, C. Kung, and J.-C. Hsu, "Dynamic analysis of a rotary hollow shaft with hot-fit part using contact elements with friction," Transactions of the Canadian Society for Mechanical Engineering, vol. 35, no. 3, 2011.

[30] E. Sikanen, J. E. Heikkinen, and J. Sopanen, "Shrink-fitted joint behavior using three-dimensional solid finite elements in rotor dynamics with inclusion of stress-stiffening effect," Advances in Mechanical Engineering, vol. 10, no. 6, Article ID 168781401878005 , June 2018.

[31] Z. Huang, B. Han, and Y. Le, "Modeling method of the modal analysis for turbomolecular pump rotor blades," Vacuum, vol. 144, pp. 145-151, 2017.

[32] Y. Zhang, K. Wang, Z. He, and L. Zhai, "Rotor-dynamic analysis for magnetic turbomolecular pump rotor with different assembly relations," in Proceedings of the 2019 22nd international conference on electrical machines and systems ICEMS, vol. 3181-3185, p. 345, August 2019.

[33] N. Zeng, Z. Wang, W. Liu, H. Zhang, K. Hone, and X. Liu, “A dynamic neighborhood-based switching particle swarm optimization algorithm," IEEE Transactions on Cybernetics, pp. 1-12, 2021.

[34] Y. Gu, Y. Liu, and C. Lu, "Brake noise reduction method based on Monte Carlo sampling and particle swarm optimization," Shock and Vibration, vol. 2021, Article ID 8878223, 11 pages, 2021.

[35] X. Lv, D. Zhou, L. Ma, Y. Zhang, and Y. Tang, “An improved Lagrange particle swarm optimization algorithm and its application in multiple fault diagnosis," Shock and Vibration, vol. 2020, Article ID 1091548, 8 pages, 2020.

[36] X. Zhang, H. Liu, and L. P. Tu, "A modified particle swarm optimization for multimodal multi-objective optimization," Engineering Applications of Artificial Intelligence, vol. 95, Article ID 103905, 2020. 